\title{
Trauma: característica sociodemográficas das vítimas e aspectos clínicos-assistenciais de sua ocorrência em hospital de urgência
}

Trauma: socio-demographic characteristics of the victims and clinical-care aspects of their occurrence in an emergency hospital

Trauma: características socio demográficas de las vítcmias y aspectos clínicos de su presencia en un hospital de urgencias

Gabriela Maria Lara de Paulo1*, Carlos Matheus Pierson Colares ${ }^{1}$, Mykaella Cristina Araújo Margarida $^{2}$, Andressa Rodrigues da Silva ${ }^{3}$, Ana Caroliny da Silva ${ }^{1}$, Lais Lara Silva Xavier ${ }^{1}$, Thainara Lorraine Costa e Silva Pereira ${ }^{1}$, Suzy Aparecida Luiz da Silva ${ }^{1}$, Thais Vilela de Sousa ${ }^{2}$, Lorena Morena Rosa Melchior².

\section{RESUMO}

Objetivo: Descrever as características sociodemográficas das vítimas de trauma bem como aspectos circunstanciais e clínico-assistenciais de suas ocorrências em um hospital de urgência referência em traumas no centro-oeste do país. Métodos: Trata-se de um estudo com abordagem quantitativa do tipo transversal descritiva. A amostra foi composta de 1148 prontuários compostos por pacientes de ambos os sexos, com idade igual ou superior a 18 anos, admitidos na sala vermelha de centro de trauma, no período de janeiro a dezembro de 2019. Resultados: A prevalência dos traumas foi em adultos jovens, com faixa etária entre 18 a 24 anos $(21,43 \%)$, do sexo masculino $(71,60 \%)$. A maioria ocorreu nos dias do final de semana e no período noturno, quanto a gravidado dos traumas, $72,47 \%$ foram classificadas como moderado. A principal causa dos traumas foram os acidentes automobilísticos $(49,47 \%)$ que, em sua maioria, envolveram motocicletas. Em $36,76 \%$ dos casos ocorreram politraumas e o sítio anatômico mais acometido foram os membros inferiores $(21,73 \%)$. Conclusão: Há uma preocupante prevalência de trauma em adultos jovens e homens, tendo como principal etiologia o transporte terrestre, com ênfase nas motocicletas. Os resultados sinalizam a necessidade de intensificar as medidas políticas, sociais e educativas.

Palavras-chave: Trauma, Urgência, Emergência.

\begin{abstract}
Objective: To describe the sociodemographic characteristics of trauma victims, as well as the circumstantial and clinical-assistance aspects of their occurrence in a reference trauma emergency hospital in the Midwest of the country. Methods: This is a study with a quantitative, descriptive, transversal approach. The study consists of 1,148 clinical histories composed by patients of both sexes, aged 18 or older, admitted to the red room of a trauma center, from January to December 2019. Results: The prevalence of trauma was in young adults, aged between 18 and 24 years $(21.43 \%)$, males $(71.60 \%)$. The majority occurred on weekends and at night, as they were embarrassed by trauma, and $72.47 \%$ were classified as moderate. The main cause of trauma was car accidents (49.47\%), which in its majority involved motorcycles. In $36.76 \%$ of cases there were multiple traumas and the most affected anatomical site on the lower limbs $(21.73 \%)$. Conclusion: There is a worrying prevalence of trauma in young adults and males, with land transport as the main etiology, with an emphasis on motorcycles. The results indicate the need to intensify political, social and educational measures.
\end{abstract}

Keywords: Trauma, Urgency, Emergency.

\footnotetext{
${ }^{1}$ Secretaria de Estado de Saúde de Goiás (SES-GO), Goiânia - GO. *E-mail: gabrielamarialara@gmail.com

2 Universidade Federal de Goiás (UFG), Goiânia - GO.

${ }^{3}$ Universidade Católica de Brasília (UCB), Brasília - DF.
}

SUBMETIDO EM: 8/2021

ACEITO EM: 8/2021

PUBLICADO EM: 10/2021 


\section{RESUMEN}

Objetivo: Describir las características sociodemográficas de las víctimas de trauma, así como los aspectos circunstanciales y clínico-asistenciales de su ocurrencia en un hospital de emergencia de referencia por trauma en el Medio Oeste del país. Métodos: Se trata de un estudio con enfoque cuantitativo, descriptivo, transversal. La muestra estuvo conformada por 1148 historias clínicas compuestas por pacientes de ambos sexos, de 18 años o más, ingresados en la sala roja de un centro de trauma, de enero a diciembre de 2019. Resultados: La prevalencia de trauma fue en adultos jóvenes, con edades entre 18 y 24 años (21,43\%), hombres $(71,60 \%)$. La mayoría ocurrieron los fines de semana y por la noche, ya que las embarazadas por traumatismo, el $72,47 \%$ se clasificaron como moderadas. La principal causa de traumatismo fueron los accidentes automovilísticos (49,47\%), que en su mayoría involucró a motocicletas. En el $36,76 \%$ de los casos hubo múltiples traumatismos y el sitio anatómico más afectado fueron los miembros inferiores $(21,73 \%)$. Conclusión: Existe una preocupante prevalencia de trauma en adultos jóvenes y hombres, con el transporte terrestre como principal etiología, con énfasis en las motocicletas. Los resultados señalan la necesidad de intensificar las medidas políticas, sociales y educativas.

Palabras clave: Trauma, Urgencia, Emergencia.

\section{INTRODUÇÃO}

Trauma é uma doença definida como lesão de dimensão, impacto e gravidade relativa, que pode ter sido provocado de forma acidental ou proposital e é capaz de gerar distúrbios locais ou sistêmicos. Essa lesão pode ser infligida por agentes mecânicos, químicos, elétricos, radioativos e térmicos (VIEIRA C, et al., 2011; NATIONAL ASSOCIATION OF EMERGENCY MEDICAL TECHNICIANS (NAEMT), 2017).

O trauma é responsável por 5,8 milhões de mortes por ano no mundo. A incidência da morte por traumas é $32 \%$ maior que a somatória dos óbitos por doenças infecciosas como malária, tuberculose e o vírus da imunodeficiência humana. As mortes traumáticas equivalem a $10 \%$ de todas as causas de óbito no mundo, e enfatiza-se a necessidade de intervenções, pois é esperado que essa taxa aumente ainda mais até 2030 (ORGANIZAÇÃO MUNDIAL DA SAÚDE (OMS), 2012; ACADEMIA BRASILEIRA DE CIÊNCIAS (ABC), 2019).

Os traumas também são responsáveis pela maior parte das incapacitações permanentes, pois a cada morte por lesões traumáticas, três pessoas ficam com sequelas permanentes, gerando gastos de aproximadamente 670 bilhões de dólares por ano com os custos médicos e a perda da produtividade (DRUMOND D, 2014).

No Brasil, no ano de 2015 a principal causa de morte entre adultos com idade entre 20 a 39 anos de ambos os sexos, foram as causas externas, sendo homicídio a primeira e acidentes com transportes terrestres a segunda que acumulou mais óbitos (MINISTÉRIO DA SAÚDE, 2018a). Os pacientes vítimas de trauma geram uma grande demanda aos serviços do sistema de saúde público, pois, muitas vítimas são encaminhadas para serem atendidas em condições de urgências e emergências. Diante disso é evidente a necessidade de implementar medidas de prevenção mais efetivas para a redução desse fenômeno (MINISTÉRIO DA SAÚDE, 2015).

A incidência, prevalência e letalidade do trauma na sociedade impacta não só em termos de custos diretos e indiretos em saúde, mas também pela incapacidade e modificação na rotina de vida das vítimas Acreditase que conhecer as características da população vítima de trauma, as características dos atendimentos dos mesmos e as características clínicas dos traumas nesse estado brasileiro irá subsidiar informações que contribuirão para o planejamento e direcionamento dos programas e políticas voltadas à prevenção deste evento no estado (DRUMOND D, 2014; ABC, 2019).

Diante disso, o objetivo do estudo foi descrever as características sociodemográficas das vítimas de traumas, bem como aspectos circunstanciais e clínico-assistenciais de suas ocorrências em um centro de trauma na região centro-oeste do país. 


\section{MÉTODOS}

Trata-se de um estudo com a abordagem quantitativa do tipo transversal descritiva. A estudo foi realizado retrospectivamente em prontuários eletrônicos pela análise de internações de janeiro a dezembro do ano de 2019, em um hospital de grande porte do centro-oeste do país que presta assistência de alta e média complexidade em urgência e emergência, com foco em traumatologia, queimaduras e medicina intensiva.

Para garantia do poder do estudo e sua validade externa, foi realizado cálculo amostral considerando o total de pacientes atendidos na emergência no ano de 2019 (42.058), dos quais 44,14\% (18.565) foram vítimas de trauma. Levou-se em consideração ainda, uma margem de erro de 5\%, nível de confiança de 99,9\%. Dessa forma, a amostra necessária calculada foi de 1042 pacientes, aos quais foram adicionados $10 \%$ com o objetivo de cobrir possíveis inconsistências, o que totalizou 1148 pacientes.

Sendo assim, a população do estudo era composta por prontuários de pacientes vítimas de trauma admitidos na ala vermelha da emergência do referido local de estudo no ano de 2019. Foram incluídos na pesquisa, prontuários de pacientes de ambos os sexos, com idade igual ou superior a 18 anos, admitidos na sala vermelha e que tiveram o diagnóstico de trauma. Foram excluídos pacientes admitidos na sala vermelha provenientes de outro setor interno do hospital, pacientes vítimas de trauma admitidos na emergência pelo serviço de triagem da própria instituição e pacientes admitidos que retornaram ao hospital devido ao mesmo trauma. Foram analisados 2642 prontuários e integraram a amostra final do estudo 1148 prontuários, como 0 apontado pelo amostral.

Ainda sobre os procedimentos de amostragem, os prontuários dos pacientes também que foram selecionados levando-se em consideração uma distribuição proporcional ao longo dos meses observados no ano de 2019, para não incorrer em viés de seleção e ocasionar uma falsa percepção de algum mês em específico, conforme a tabela a seguir (Tabela 1). Os prontuários também foram selecionados, mediante sorteio, garantindo que a amostra fosse determinada de forma aleatória.

Tabela 1 - Distribuição proporcional dos pacientes com trauma atendidos na emergência considerando os meses do ano de 2019.

\begin{tabular}{lccc}
\hline \multicolumn{1}{c}{ MÊS } & NÚMERO DE TRAUMAS & $\%$ & AMOSTRA \\
\hline Janeiro & 1659 & 8,94 & 102 \\
Fevereiro & 1428 & 7,69 & 88 \\
Março & 1536 & 8,27 & 96 \\
Abril & 1469 & 7,91 & 91 \\
Maio & 1540 & 8,30 & 95 \\
Junho & 1399 & 7,54 & 87 \\
Julho & 1584 & 8,53 & 98 \\
Agosto & 1448 & 7,80 & 89 \\
Setembro & 1580 & 8,51 & 98 \\
Outubro & 1678 & 9,04 & 104 \\
Novembro & 1622 & 8,74 & 100 \\
Dezembro & 1622 & 8,74 & 100 \\
\hline Total & $\mathbf{1 8 . 5 6 5}$ & $\mathbf{1 0 0 , 0 0}$ & $\mathbf{1 1 4 8}$ \\
\hline
\end{tabular}

Fonte: Paulo GML, et al., 2021.

A coleta de dados foi realizada entre abril a agosto de 2019 mediante a análise retrospectiva dos prontuários dos pacientes, sendo estes disponíveis na instituição na forma eletrônica. A coleta foi subsidiada por um instrumento de pesquisa semiestruturado desenvolvido pela equipe de pesquisadores com variáveis como idade, sexo, cidade de origem dos pacientes; turno do dia, dia da semana e mês que ocorreu; gravidade do trauma, etiologia do trauma, sítio anatômico do trauma, tipo de lesão, necessidade de procedimento cirúrgico e desfecho do caso. 
Para apresentação dos dados, foi realizada análise descritiva utilizando o software STATA® versão 14.0 e foram explicitados por frequência absoluta e frequência relativa para as variáveis categóricas e para as variáveis contínuas, utilizou-se média e desvio padrão.

A pesquisa seguiu as normas e diretrizes que regulamentam as pesquisas que envolvem seres humanos e as determinações contidas na Resolução 466/2012, do Conselho Nacional de Saúde. Esse estudo foi aprovado pelo comitê de ética em pesquisa Leide das Neves Ferreira sob número de parecer: 3.974.153. Por se tratar de um estudo de dados secundários, foi concedida a isenção da assinatura do termo de consentimento e livre esclarecimento, foi assinado o termo de compromisso para a utilização e manuseio de dados.

\section{RESULTADOS}

Obteve-se uma amostra de 1148 prontuários de pacientes vítimas de trauma, a média de idade foi de 39,88 anos, com desvio padrão de 17-79. Houve uma prevalência de traumas em adultos jovens, com idade entre 18 a 24 anos (21.43\%), e a maioria da amostra 822 (71.60\%) do sexo masculino. Em relação a região de residência desses pacientes, 681 (59.32\%) residia na cidade do hospital em estudo. Dentre os 467 (40.68\%) pacientes encaminhados para instituição, proveniente de outras cidades, a maioria $386(82.65 \%)$ eram de cidades do centro do estado (Tabela 2).

Tabela 2 - Distribuição dos casos de trauma, segundo dados sociodemográficos ( $n=1148)$.

\begin{tabular}{|c|c|c|}
\hline Variáveis & $\mathbf{n}$ & $\%$ \\
\hline \multicolumn{3}{|l|}{ Faixa etária } \\
\hline 18 a 24 anos & 246 & 21,43 \\
\hline 25 a 31 anos & 228 & 19,86 \\
\hline 32 a 38 anos & 177 & 15,42 \\
\hline 39 a 45 anos & 135 & 11,76 \\
\hline 46 a 52 anos & 106 & 9,23 \\
\hline 53 a 59 anos & 90 & 7,84 \\
\hline 60 a 66 anos & 50 & 4,36 \\
\hline 67 a 73 anos & 40 & 3,48 \\
\hline 74 a 80 anos & 33 & 2,87 \\
\hline 81 anos ou mais & 43 & 3,75 \\
\hline \multicolumn{3}{|l|}{ Sexo } \\
\hline Masculino & 822 & 71,60 \\
\hline Feminino & 324 & 28,22 \\
\hline Não informado & 2 & 0,17 \\
\hline \multicolumn{3}{|l|}{ Região de Origem } \\
\hline Capital do Estado & 681 & 59,32 \\
\hline Interior do Estado & 454 & 39,55 \\
\hline Outro Estado & 13 & 1,13 \\
\hline \multicolumn{3}{|c|}{ Região de Origem do Estado } \\
\hline Centro & 386 & 85,02 \\
\hline Sul & 37 & 8,15 \\
\hline Noroeste & 26 & 5,73 \\
\hline Leste & 3 & 0,66 \\
\hline Norte & 2 & 0,44 \\
\hline
\end{tabular}

Fonte: Paulo GML, et al., 2021.

A maioria dos pacientes $832(72,47 \%)$ foram classificados quanto à gravidade do trauma, em moderado; $207(18,03 \%)$ em grave e os demais como trauma leve ou não informado. Em relação aos dias da semana em que ocorreram o trauma, na segunda e terça houveram $165(14,3 \%)$ traumas, quarta $155(13,5 \%)$ traumas, quinta $136(11,8 \%)$ traumas, sexta $146(12,7 \%)$ traumas, sábado $181(15,7 \%)$ traumas e no domingo 200 $(17,4 \%)$ traumas. Sucedendo uma uniformidade da amostra durante os dias da semana, com um aumento no número de casos nos finais de semana 
Quanto à etiologia do trauma, a maioria 568 (49.47\%) foi proveniente acidentes automobilísticos seguidos pelas quedas com 202 (17.59\%) casos, com pico de prevalência no mês de julho (Gráfico 1). Desses, 427 (75.70\%) ocorreram em acidentes com motocicletas, seguido 96 (17.03\%) carro, 31 (5.49\%) bicicleta e os demais meios de transporte somaram 10 (1.78\%).

Gráfico 1 - Casos de traumas de acordo com a etiologia e o mês do ocorrido.

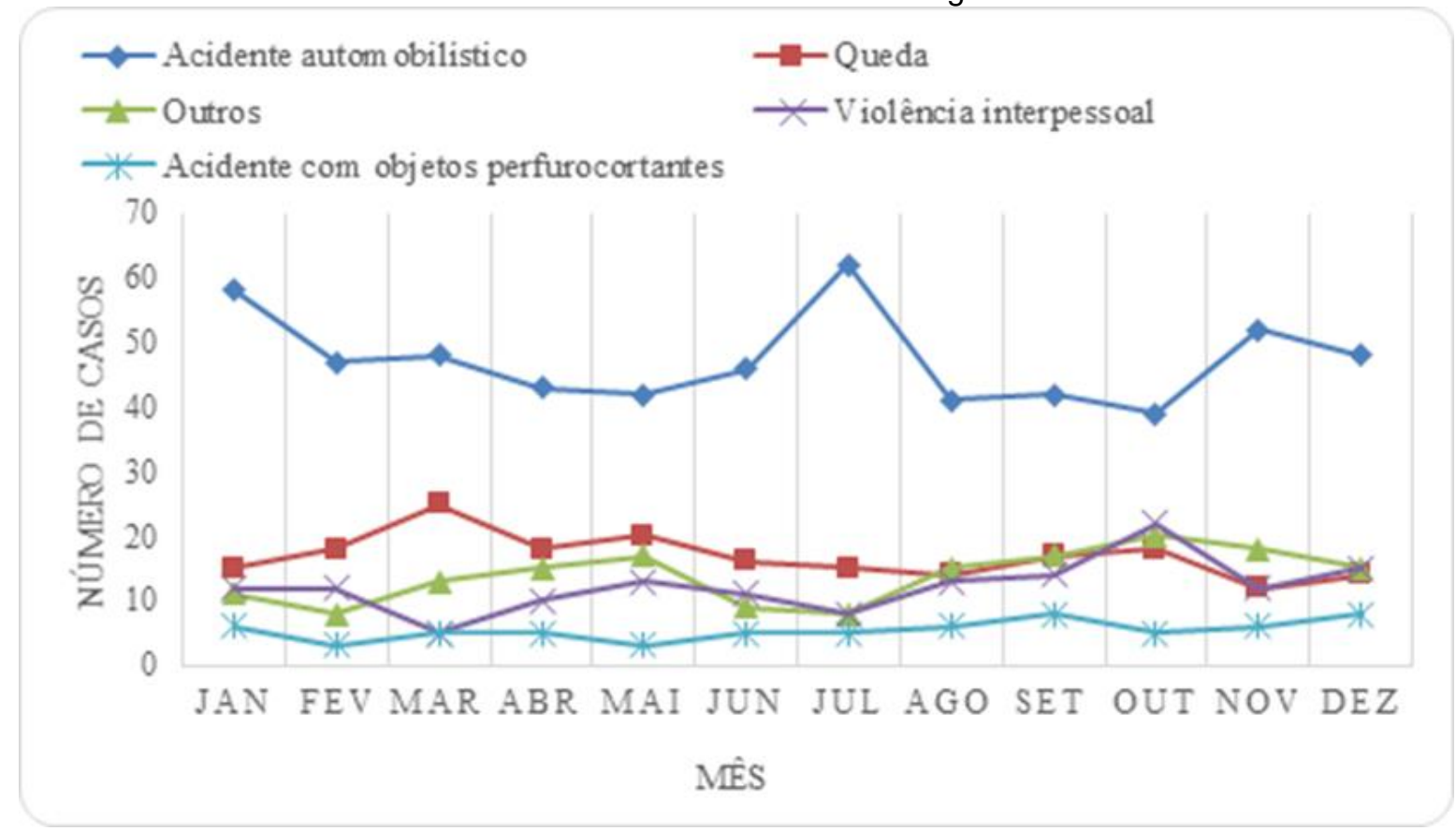

Fonte: Paulo GML, et al., 2021.

O período noturno teve maior número de casos 495 (43,12\%), seguido pelo período vespertino com 343 $(29,88 \%)$ e o período matutino com 310 (27,00\%). Tendo um pico de prevalência de acidentes automobilísticos e de violência interpessoal aos domingos à noite (Gráfico 2 e Gráfico 3).

Gráfico 2 - Casos de traumas de acordo com a etiologia e o dia da semana.

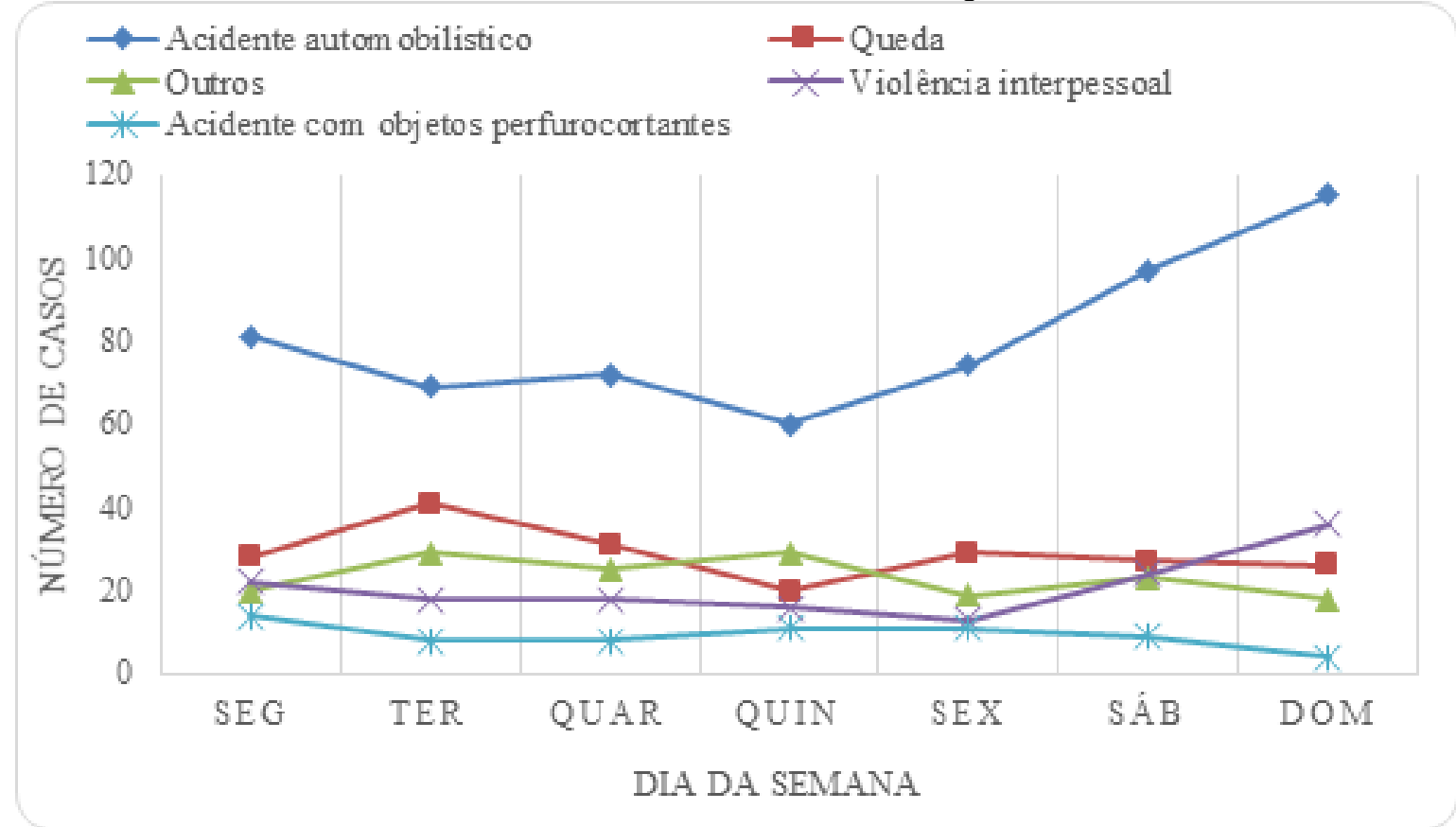

Fonte: Paulo GML, et al., 2021. 
Gráfico 3 - Casos de traumas de acordo com a etiologia e o turno.

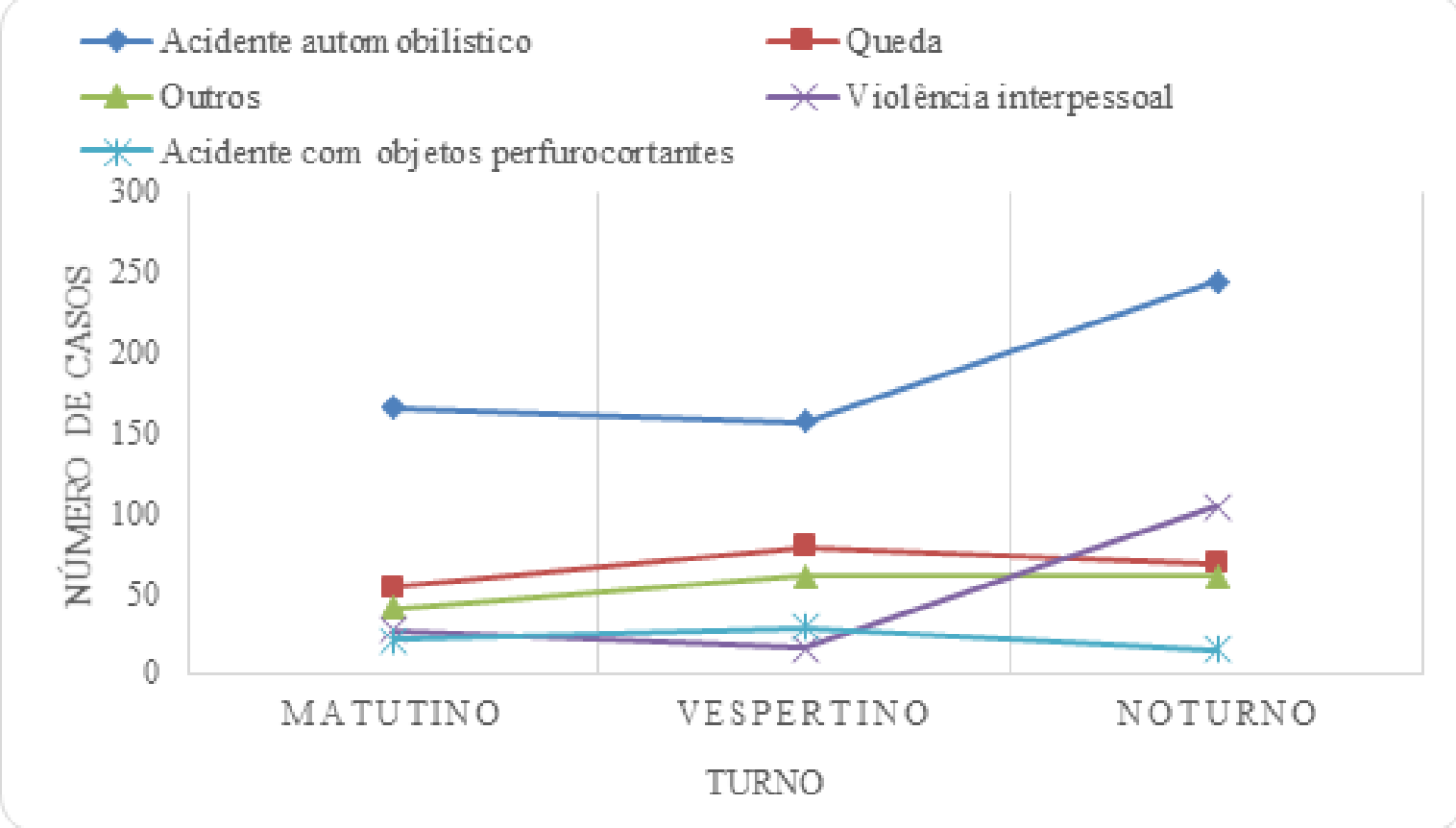

Fonte: Paulo GML, et al., 2021.

Dentre toda a amostra ocorreram 422 casos (36,76\%) de politraumas, sendo o sítio anatômico mais acometido por traumas foram os membros inferiores com 381 casos $(21,73 \%)$, seguido pelos membros superiores com 327 casos (18,53\%). As fraturas ocorreram em 472 dos casos $(41,11 \%)$, sendo 138 fraturas expostas (12,02\%) e 334 casos de fraturas fechadas $(29,09 \%)$.

As cirurgias devido aos traumas foram realizadas em $385(33,53 \%)$ dos casos, onde $271(23,61 \%)$ pacientes foram submetidos a cirurgias de emergência, 21 (5,45\%) foram submetidos a cirurgias de urgência e $93(24,15 \%)$ foram submetidos a cirurgias eletivas.

O principal desfecho imediato encontrado nos pacientes vítimas de trauma que deram entrada na sala vermelha foi a alta hospitalar em $548(74.74 \%)$ pacientes. Foi encontrada uma taxa de óbito de $39(3.40 \%)$ pacientes (Tabela 3).

Tabela 3 - Distribuição dos casos de trauma, segundo o desfecho dos casos.

\begin{tabular}{lcccc}
\hline \multirow{2}{*}{ Variável } & \multicolumn{2}{c}{ Desfecho imediato } & \multicolumn{2}{c}{ Desfecho tardio } \\
\cline { 2 - 5 } & $\mathbf{n}$ & $\%$ & $\mathbf{n}$ & $\%$ \\
\hline Alta hospitalar & 548 & 47,74 & 484 & 42,16 \\
Centro cirúrgico & 271 & 23,61 & - & - \\
Internação na enfermaria & 186 & 16,20 & - & - \\
Observação no pronto socorro & 42 & 3,66 & - & - \\
Internação na UTI & 37 & 3,22 & - & - \\
Não informado & 23 & 2,00 & 1 & 0,09 \\
Evasão & 20 & 1,74 & 6 & 0,52 \\
Transferência para outra unidade & 13 & 1,13 & 14 & 1,22 \\
Óbito & 8 & 0,70 & 31 & 2,70 \\
\hline
\end{tabular}

Nota: As informações ausentes no desfecho tardio se justificam pelo fato que nessa situação o paciente somente poderia evoluir para alta hospitalar, evasão, transferência para outra unidade ou óbito.

Fonte: Paulo GML, et al., 2021.

\section{DISCUSSÃO}

No presente estudo foram identificados a ocorrência de 18.565 casos de trauma atendidos na sala vermelha do pronto socorro do hospital investigado. $O$ trauma dentre as causas externas, é a principal causa de óbito entre pessoas jovens, sendo que no ano de 2016, 4,9 milhões de pessoas morreram em 
consequência desses agravos. Mundialmente, as lesões causadas em acidentes com transportes terrestres são a oitava causa de morte e decorrente desses traumas 1,4 milhões de pessoas foram a óbito (WORLD HEALTH ORGANIZATION (WHO), 2018; LENTSCK M, et al., 2019). Esses dados já demonstram o quanto é necessário intensificar as medidas de prevenção dos traumas e melhorar o atendimento aos mesmos.

Como resultado desse estudo observou-se que a média de idade dos pacientes foi de 39,88 anos, resultado que foi menor quando comparado a literatura (VIEL I, et al., 2019). Também com uma frequência maior de traumas em adultos jovens e na sua maioria em pessoas do sexo masculino. Esses resultados se assemelham com estudos nacionais e internacionais (BYUN C, et al., 2015; WHO, 2018; VIEL I, et al., 2019).

Os jovens adultos sofrem mais traumas devido ao estilo de vida e processo cultural que esse grupo está inserido. Desde que a Política Nacional de Saúde do Homem foi lançada em 2009, o Ministério da Saúde do Brasil já sinalizava que devido a brutalidade que está ligado aos homens, ao maior uso de bebidas alcoólicas, o uso de drogas e maior acessos a armas, eles estavam mais propensos a sofrer mais traumas (MINISTÉRIO DA SAÚDE 2009a; ALVES R, et al., 2012).

Em relação a distribuição de pacientes que residem na capital e região metropolitana, pode-se associar ao fato dessas regiões serem as mais populosas do estado. Apenas nesta capital brasileira tem uma população de 1.446 .106 habitantes, o que é $28,90 \%$ de toda população do estado (INSTITUTO MAURO BORGES DE ESTATÍSTICAS E ESTUDOS SOCIOECONÔMICOS (IMB), 2018). Contudo, o número de casos provenientes do interior do estado é expressivo e pode ser justificado pela demanda que os casos mais graves têm de serem encaminhados para um hospital especializado que tenha estrutura para oferecer os cuidados necessários (VIEL I, et al., 2019).

Os resultados do presente estudo se assemelham com o estudo realizado na Índia, onde os pacientes vítimas de trauma, na sua maioria são classificados como gravidade moderada. Ao ser admitido no hospital, o paciente é submetido à classificação de risco para identificar a gravidade e a urgência de cada caso e deve ser realizado pelo profissional capacitado. Por se tratar da ala vermelha do pronto socorro de um serviço de saúde de alta complexidade, era esperado que a maioria dos casos fossem classificados como moderados e graves, pois por definição, essa área é destinada para pacientes que necessitam de um atendimento imediato devido ao risco do caso se agravar ou em virtude da necessidade da realização de procedimentos invasivos (MINISTÉRIO DA SAÚDE, 2009b; ABHILASH K, et al., 2019).

Os acidentes automobilísticos foram a primeira causa de traumas nesse estudo, sendo a sua maioria acidentes com motocicletas; também foi identificado que, traumas causados por violência interpessoal, que incluem agressões por arma branca, agressões com arma de fogo e agressões físicas foram a $4^{\circ}$ causa de traumas. Esses resultados corroboram com outras pesquisas realizadas em 2019, no âmbito nacional e internacional (ABHILASH K, et al., 2019; SOUZA A, et al., 2019). Porém diverge de alguns resultados encontrados, em outros estudos onde a principal etiologia foi a queda (SILVA L, et al., 2017; VIEL I, et al., 2019). Essa divergência com algumas pesquisas pode ser atribuída ao método desses estudos pois foram feitos em hospitais que não são centro de trauma ou devido a faixa etária da população estudada.

Mundialmente, as lesões causadas em acidentes com transportes terrestres são a oitava causa de morte. No ano de 2016, 1,4 milhões de pessoas morreram em decorrência de acidentes automobilísticos. A taxa de óbitos devido a acidentes de trânsito em países de baixa renda é maior do que a taxa em países de alta renda. A taxa dos países de baixa renda é de 29,4 mortes por 100.000 habitantes, a taxa mundial é de 18,8 mortes por 100.000 habitantes (WHO, 2018).

São múltiplos os fatores que contribuem para os acidentes de trânsito, em especial, os que envolvem motocicletas, como sexo, faixa etária, experiência em dirigir, turno do dia e o abuso de substâncias que afetam reflexos fundamentais para conduzir veículos. Estudos apontam que homens, jovens adultos estão mais propensos a esses acidentes devido aos hábitos de vida como dirigir sob influência de álcool, não obedecem às leis de trânsito como respeitar limites de velocidades, usar dispositivos de segurança e uso de objetos que distraem a atenção como telefone (MINISTÉRIO DA SAÚDE 2019; MONTEIRO C, et al., 2020). 
No estado brasileiro onde ocorreu o estudo, a violência interpessoal e lesões no trânsito foram a $6^{\underline{a}}$ e $7^{\text {a }}$ causas de morte respectivamente no ano de 2015. Tendo uma prevalência de 34,6 óbitos por 100 mil habitantes em relação a violência interpessoal e de 32,5 óbitos por 100 mil habitantes por acidentes no trânsito (MINISTÉRIO DA SAÚDE, 2018a).

Ainda sobre os traumas causados por violência interpessoal, que aumentam nos finais de semana e no período noturno, quando comparado aos outros dias e turno pode ser devido às violências urbanas fomentadas pela desigualdade social, a marginalização de alguns grupos e crises econômicas que propiciam o tráfico de drogas e o crime organizado. É fundamental que medidas para reduzir os índices de violência sejam implementadas pois, altas taxas de violência influenciam no desenvolvimento socioeconômico da população e indicadores de saúde. Para reduzir a violência de uma região, é importante que sejam desenvolvidos projetos e políticas que tenham como objetivo o desenvolvimento social (MELO A e GARCIA L, 2019).

Em relação às quedas, elas ocorrem devido a uma soma de vários aspectos, como idade, ambiente, condições médicas pré-existentes, uso de medicamentos ou substâncias e profissão. Os acidentes de causa externa dessa natureza, são muitas vezes banalizados, por serem considerados de baixa energia cinética. Porém elas podem causar traumas graves, como as fraturas que podem pôr em risco a vida da vítima (SILVA L, et al., 2017).

O achado de que os sítios anatômicos mais acometidos nos traumas foram os membros e que o principal tipo de lesão foram as fraturas, coaduna com a literatura (BYUN C, et al., 2015; DIMAGGIO C, et al., 2016; SILVA L, et al., 2017). A maior distribuição dos traumas musculoesqueléticos em membros pode ser justificada por essas partes do corpo estarem mais expostas, o que propicia um maior número de traumas. Essa categoria de trauma é mais desfavorável para a saúde pública e para os pacientes do que os outros traumas pois, o tempo de recuperação é longo, tem alta taxa de letalidade principalmente entre os idosos e muitas das vezes deixa sequelas, o que impede os indivíduos a retornarem às suas atividades laborais (SILVA L, et al., 2017; AUGUSTO V, et al., 2018; DAMASCENO I, et al., 2018).

No que tange às cirurgias de emergência, esse procedimento se mostrava necessário em até 24 horas para reverter um risco iminente à vida, órgão ou tecido devido ao trauma sofrido. De acordo com os resultados é possível afirmar que a maioria das cirurgias foram em membros inferiores. Com esse e outros estudos vêse alta ocorrência de fraturas nos traumas que acometem essa parte do corpo e que na maioria das vezes há indicação para intervenção cirúrgica. Logo, essas lesões são capazes de colocar o paciente em risco de perder o membro ou a vida, pois descontinuidade óssea pode desencadear o principal tipo de choque relacionado aos traumas, o hipovolêmico, onde há uma perda excessiva de sangue. A conduta para essa situação é realizar a reposição volêmica e estancar o sangramento. Nesses casos, o procedimento cirúrgico imediato é primordial para reverter o agravamento do caso clínico (MAVROGENES A, 2016; AMERICAN COLLEGE OF SURGEONS, 2018; SILVA I, et al., 2017).

O desfecho em sua maioria foi a alta hospitalar, das quais $47,74 \%$ tiveram alta imediata. Resultados semelhantes foram encontrados em estudo realizado em Brasília. Essa conclusão dos casos sugere que 0 tratamento realizado no pronto socorro foi eficaz e não foi preciso ficar em observação ou de intervenção cirúrgica, o que demonstra, além de ser um desfecho positivo, uma assistência efetiva e de qualidade (ABHILASH K, et al., 2019; SOUZA A, et al., 2019).

Esse aspecto assistencial se confirma pela baixa taxa de óbito, com um total de $3,40 \%$ dos casos. Estudo realizado na Suécia identificou uma diminuição de até $41 \%$ no risco de mortalidade nos pacientes de trauma quando atendidos em centros de trauma, pois esses serviços especializados oferecem 0 atendimento adequado que os casos de traumatologia requerem (CANDEFJORD S, et al., 2020).

Diante disso, o trauma se configura como um problema para a saúde pública, pois ocasiona consequências para o paciente traumatizado, a sociedade e o sistema de saúde. Além das lesões, o trauma pode acarretar óbitos e incapacidade física, seja permanente ou transitória, e possui um alto custo com a recuperação e reabilitação do doente (LÔBO GC, et al., 2021, SANTOS LFS, et al., 2016). 
Este estudo tem limitações próprias de seu desenho metodológico, entretanto, além de ter poder estatístico assegurado pelo seu cálculo amostral e amostra atingida, possibilitou melhor compreensão da realidade de saúde traumatológica da região investigada. Esses dados também proporcionam ampla discussão a respeito da temática uma vez que os resultados do presente estudo se somam aos da literatura, constituem evidências e subsídio para políticas públicas mais assertivas e eficazes.

\section{CONCLUSÃO}

A maioria dos traumas se deu em adultos jovens, do sexo masculino, classificados como de gravidade moderada, que ocorreram no período noturno e nos finais de semana. A principal etiologia dos acidentes foram os automobilísticos, sobretudo os envolvendo motocicletas e o mês que mais ocorreram esse tipo de trauma foi em julho. Ocorreram 422 casos de politraumas e as partes do corpo mais acometidas foram os membros. Foi necessário intervenção cirúrgica, a maior parte das cirurgias foram de emergência. $O$ principal desfecho dos traumas foi a alta hospitalar. Tais resultados são relevantes pois podem ser utilizados para embasar a elaboração e direcionamento de ações políticas, sociais e educativas, visando a prevenção de acidentes automobilísticos e violência.

\section{REFERÊNCIAS}

1. ACADEMIA BRASILEIRA DE CIÊNCIAS (ABC). Trauma é uma doença: um apelo à ação da IAP Health. Academia Brasileira de Ciências. Brasil, 2019. Disponível em: https://www.interacademies.org/news/trauma-disease-call-actioniap-health.Acesso em: : 25 nov de 2020.

2. ABHILASH K, et al. Profile of trauma patients in the emergency department of a tertiary care hospital in South India. Journal of Family Medicine and Primary Care. Journal of family medicine and primary care, 2016: 5(3).

3. ALVES R, et al. Homens, vítimas e autores de violência: A corrosão do espaço público e a perda da condição humana. Interface: Communication, Health, Education, 2012: 16(43).

4. AMERICAN COLLEGE OF SURGEONS. Advanced Trauma Life Support (ATLS). 10 ed. Estados Unidos: The Committee of Trauma, 2018; 474p.

5. AUGUSTO V, et al. Incapacidade em trabalhadores com lesões musculoesqueléticas de membros superiores atendidos num centro de reabilitação. REFACS, 2018; 2 .

6. BYUN C, et al. Epidemiology of trauma patients and analysis of 268 mortality cases: trends of a single center in KOREA. Yonsei Medical Journal, 2015: 56(1).

7. CANDEFJORD S, et al. Mortality of trauma patients treated at trauma centers compared to non-trauma centers in Sweden: a retrospective study. Eur J of Trauma and Emerg Surg. 2020.

8. DAMASCENO I, et al. Caracterização clínica e epidemiológica de pacientes vítimas de acidentes motociclísticos. Enfermagem em Foco, 2018: 9(2).

9. DIMAGGIO C, et al. Traumatic Injury in the United States: In-Patient Epidemiology 2000-2011. Injury, 2016: 47(7).

10. DRUMOND D. Trauma é uma doença. Revista Médica de Minas Gerais, 2014: 24(4).

11. INSTITUTO MAURO BORGES DE ESTATÍSTICAS E ESTUDOS SOCIOECONÔMICOS (IMB). Goiás em dados 2017. Goiânia: SEGPLAN; 2018. Disponível em: https://www.imb.go.gov.br/files/docs/publicacoes/goias-emdados/godados2017.pdf. Acesso em: 01 de Dez de 2019.

12. LENTSCK M, et al. Panorama epidemiológico de dezoito anos de internações por trauma em UTI no Brasil. Revista Saúde Pública, 2019: 53(83).

13. LÔBO GC, et al. Perfil epidemiológico dos pacientes vítimas de trauma atendidos no município de Vitória da Conquista entre os anos de 2017 e 2018. Revista Eletrônica Acervo Saúde, 2021: 13(3).

14. MAVROGENIS A, et al. Vascular Injury in Orthopedic Trauma. Orthopedics, 2016: 39(4).

15. MELO A, GARCIA L. Fatores associados a agressões por desconhecidos entre jovens do sexo masculino atendidos em serviços de urgência e emergência: estudo de casos e controles. Ciência \&. saúde coletiva, 2019: 24(8).

16. MINISTÉRIO DA SAÚDE. Política Nacional de Atenção Integral à Saúde do Homem: princípios e diretrizes; Secretaria de Atenção à Saúde, Departamento de Ações Programáticas e Estratégicas. Brasília- DF, Brasil, 2009a. Disponível em: https://bvsms.saude.gov.br/bvs/publicacoes/politica_nacional_atencao_homem.pdf. Acesso em: 25 nov de 2020.

17. MINISTÉRIO DA SAÚDE. Acolhimento e classificação de risco nos serviços de urgência. Brasília - DF, Brasil, $2009 \mathrm{~b}$. Disponível em: https://bvsms.saude.gov.br/bvs/publicacoes/acolhimento_classificaao_risco_servico_urgencia.pdf. Acesso em: 25 nov de 2020.

18. MINISTÉRIO DA SAÚDE. Saúde Brasil 2014: uma análise da situação de saúde e das causas externas / Ministério da Saúde, Secretaria de Vigilância em Saúde, Departamento de Vigilância de Doenças e Agravos Não Transmissíveis e Promoção da Saúde. Brasília- DF, Brasil, 2015. Disponível em: https://bvsms.saude.gov.br/bvs/publicacoes/saude_brasil_2014_analise_situacao.pdf. Acesso em: 25 nov de 2020. 
19. MINISTÉRIO DA SAÚDE. Saúde Brasil Estados 2018: uma análise de situação de saúde segundo o perfil de mortalidade dos estados brasileiros e do Distrito Federal; Ministério da Saúde, Secretaria de Vigilância em Saúde, Departamento de Vigilância de Doenças e Agravos Não Transmissíveis e Promoção da Saúde. Brasília - DF, Brasil, 2018a. Disponível em: https://bvsms.saude.gov.br/bvs/publicacoes/saude_brasil_estados_2018_analise_situacao_saude_mortalidade.pdf. Acesso em: 25 nov de 2020.

20. MINISTÉRIO DA SAÚDE. Saúde Brasil 2017: uma análise da situação de saúde e os desafios para o alcance dos objetivos de desenvolvimento sustentável/ Ministério da Saúde, Secretaria de Vigilância em Saúde, Departamento de Vigilância de Doenças e Agravos não Transmissíveis e Promoção da Saúde. Brasília - DF, Brasil, 2018b. Disponível em:

https://bvsms.saude.gov.br/bvs/publicacoes/saude_brasil_2017_analise_situacao_saude_desafios_objetivos_desenv olvimento_sustetantavel.pdf. Acesso em: 25 nov de 2020.

21. MINISTÉRIO DA SAÚDE. Saúde Brasil 2018 uma análise de situação de saúde e das doenças e agravos crônicos: desafios e perspectivas; Ministério da Saúde, Secretaria de Vigilância em Saúde, Departamento de Vigilância de Doenças e Agravos Não Transmissíveis e Promoção da Saúde. Brasília - DF, Brasil, 2019. Disponível em: https://bvsms.saude.gov.br/bvs/publicacoes/saude_brasil_2018_analise_situacao_saude_doencas_agravos_cronico S_desafios_perspectivas.pdf. Acesso em: 25 nov de 2020.

22. MONTEIRO C, et al. Características de acidentes e padrões de lesões em motociclistas hospitalizados: estudo retrospectivo de emergência. Acta Paulista de Enfermagem, 2020: 33.

23. NATIONAL ASSOCIATION OF EMERGENCY MEDICAL TECHNICIANS (NAEMT). PHTLS - Atendimento Préhospitalizado ao Traumatizado. 8. ed. Jones \& Bartlett Learning: Estados Unidos, 2017; 744p.

24. ORGANIZAÇÃO MUNDIAL DA SAÚDE (OMS). Traumas matam mais que malária, tuberculose e AIDS, alerta OMS. Brasil: OMS, 2012. Disponível em: https://nacoesunidas.org/traumas-matam-mais-que-malaria-tuberculose-e-aidsalerta-oms/. Acesso em: 01 de Dez de 2019.

25. SANTOS LFS, et al. Estudo epidemiológico do trauma ortopédico em umserviço público de emergência. Caderno saúde coletiva, 2016; 24(4).

26. SILVA L, et al. Análise retrospectiva da prevalência e do perfil epidemiológico dos pacientes vítimas de trauma em um hospital secundário. Rev Med (São Paulo), 2017: 96:4.

27. SOUZA A, et al. Perfil epidemiológico e clínico de pacientes adultos jovens admitidos na sala amarela do centro de trauma do hospital de base do distrito Federal. Revista de Divulgação Científica Sena Aires, 2019: 8(1).

28. VIEIRA C, et al. Protocolo Clínico sobre Trauma. Belo Horizonte: Secretaria de Estado de Saúde de Minas Gerais, 2011.

29. VIEL I, et al. Factors Associated With Interhospital Transfer of Trauma Victims. Journal of Trauma Nursing, 2019: 26(5).

30. WORLD HEALTH ORGANIZATION (WHO). The top 10 causesofdeath. World Health Organization: Geneva, 2018. Disponível em: https://www.who.int/news-room/fact-sheets/detail/the-top-10-causes-of-death. Acesso em: $01 \mathrm{de}$ Dez de 2019. 